\title{
Acute Acalculous Cholecystitis after Measles
}

\author{
Emine Füsun Karaşahin, $\mathrm{MD}^{1 *}$; Ömer Karaşahin, $\mathrm{MD}^{2}$ \\ ${ }^{1}$ Erzurum Public Health Directorate, Public Health, Erzurum, Turkey \\ ${ }^{2}$ Erzurum Regional Training and Research Hospital, Clinic of Infectious Diseases, Erzurum, Turkey
}

\begin{abstract}
The recent increase in measles cases will result in a higher incidence of associated complications. Hepatobiliary complications are among rare complications of measles. Here, we present a case of measles complicated by acute acalculous cholecystitis (AAC), which we believe to be only the second case reported in the literature. A 17-year-old woman presented with a maculopapular rash, high fever, sore throat, runny nose, nausea, and generalized body aches. Measles was diagnosed by anti-measles immunoglobulin M (IgM) positivity and a 4-fold increase in immunoglobulin G (IgG) 2 weeks later. On day 5, Murphy's sign was positive and antibiotic therapy was initiated. Two days later, laparoscopic cholecystectomy was performed due to persistent fever and abdominal pain. The pathology result was consistent with nonspecific cholecystitis. As this case highlights, it should be kept in mind that AAC, which is a rare complication of the multisystem involvement caused by measles, can occur during recovery from the infection. Keywords: Acalculous cholecystitis, Complications, Hepatobiliary, Measles

Cite this article as: Karaşahin EF, Karaşahin O. Acute acalculous cholecystitis after measles. Arch Iran Med. 2021;24(6):505-507. doi: 10.34172/aim.2021.72
\end{abstract}

Received: January 24, 2021, Accepted: March 15, 2021, ePublished: June 1, 2021

\section{Introduction}

Acute-onset measles (also known as rubeola) is a highly contagious infectious disease caused by the Measles morbillivirus $(\mathrm{MeV})$ and is usually seen in children. It is characterized by fever, cough, coryza, conjunctivitis, malaise, and a maculopapular rash that appears a few days after the onset of initial symptom. Pathognomonic Koplik's spots may form in the buccal mucosa 2 to 3 days before the typical measles rash. ${ }^{1}$

Measles often resolves without complications, but approximately $30 \%$ of patients develop one or more complications. ${ }^{1,2}$ Because the virus causes immunosuppression by targeting epithelial, reticuloendothelial, and white blood cells (WBCs), the complications of measles have been reported in every organ system. ${ }^{3}$ Giant cells characteristic of measles can be detected by immunological staining of stomach or appendix biopsies. ${ }^{1,3}$ The most common complications of measles are those affecting the respiratory tract (otitis media, mastoiditis, pneumonia, etc) and central nervous system (encephalitis, subacute sclerosing pan encephalitis, Guillain-Barre syndrome). ${ }^{2}$ Reports of hepatic complications during measles infection have also been reported but are rare. ${ }^{4,5}$ Diarrhea, mesenteric adenitis, hepatitis, and pancreatitis can be counted among gastroenterological complications. ${ }^{2}$

In this report, we present a case of acute acalculous cholecystitis (AAC) associated with measles, which, to the best of our knowledge, has only been documented once before in the literature.

\section{Case Report}

A 17-year-old woman presented to the dermatology clinic due to a maculopapular rash that first appeared on her face 2 days earlier and then spread to her trunk and upper extremities. She also reported complaints of fever, sore throat, runny nose, nausea, and generalized body aches and was referred to the infectious diseases clinic. Physical examination revealed pharyngitis, and cervical and axillary lymphadenopathy. The maculopapular rash covered her face, upper body, and upper extremities. Her medical history was unremarkable except that she had not received childhood vaccinations. Abdominal examination was normal. The patient was hospitalized to investigate the etiology of her fever and maculopapular rash.

Negative results were obtained in group A streptococcus rapid antigen test, Rubella immunoglobulin $\mathrm{M}(\operatorname{IgM})$ and immunoglobulin G (IgG), Epstein-Barr virus viral capsid antigen (VCA) IgM, cytomegalovirus IgM, parvovirus IgM, anti-HIV, anti-HAV IgM, anti-HCV, anti-HBc IgM, Zika virus and Chikungunya virus reverse transcriptasepolymerase chain reaction (RT-PCR), and Brucella tube agglutination and Salmonella group agglutination tests. No microorganisms were detected in throat culture, blood culture (4 sets), or urine culture. On admission, C-reactive protein (CRP) was $65.3 \mathrm{mg} / \mathrm{L}$, alanine aminotransferase (ALT) was $30 \mathrm{U} / \mathrm{L}$, aspartate aminotransferase (AST) was $25 \mathrm{U} / \mathrm{L}$, gamma glutamyl transferase (GGT) was $24 \mathrm{U} / \mathrm{L}$, platelet count was $119 \times 10^{9} / \mathrm{L}$, WBC count was $8.64 \times$ $10^{9} / \mathrm{L}$, and lymphocyte count was $0.47 \times 10^{9} / \mathrm{L}$. The measles diagnosis was confirmed by anti-MeV IgM positivity and 
a 4 -fold increase in anti-MeV IgG after 2 weeks (Figure 1). The samples were analyzed in the microbiology laboratory using the Micro-ELISA method (ALISEI SEAC, Italy).

On day 3 of hospitalization, the rash faded and fever subsided. Laboratory parameters were: CRP $32.4 \mathrm{mg} / \mathrm{L}$, ALT $25 \mathrm{U} / \mathrm{L}$, AST $27 \mathrm{U} / \mathrm{L}$, GGT $24 \mathrm{U} / \mathrm{L}$, platelet count 142 $\times 10^{9} / \mathrm{L}$, WBC count $9.81 \times 10^{9} / \mathrm{L}$, and lymphocyte count $1.51 \times 10^{9} / \mathrm{L}$.

On day 5, the patient's fever recurred. She reported abdominal pain and Murphy's sign was positive on physical examination. On abdominal ultrasound (US), gallbladder wall thickness was at the upper limit of normal at $5 \mathrm{~mm}$ and the gallbladder was surrounded by a $2-\mathrm{mm}$ thick layer of free fluid. Intraluminal calculus was not detected. Laboratory parameters on day 5 were: CRP $66.4 \mathrm{mg} / \mathrm{L}$, ALT $68 \mathrm{U} / \mathrm{L}$, AST $50 \mathrm{U} / \mathrm{L}$, GGT $78 \mathrm{U} / \mathrm{L}$, platelet count $198 \times 109 / \mathrm{L}$, WBC count $14.63 \times 109 / \mathrm{L}$, and lymphocyte count $2.08 \times 10^{9} / \mathrm{L}$. Oral intake was discontinued and parenteral fluid therapy was initiated with intravenous (IV) ceftriaxone $1 \mathrm{~g}$ twice a day. After 48 hours of antibiotic therapy, laparoscopic cholecystectomy was performed due to persistent abdominal pain and fever. The pathology report stated that the gallbladder material measured $6 \times 4 \times 3 \mathrm{~cm}$ and appeared hydropic; the reported diagnosis was "nonspecific cholecystitis". The patient did not have abdominal pain postoperatively.

Laboratory parameters evaluated before discharge were: CRP < 3.2 mg/L, ALT 17 U/L, AST 20 U/L, GGT 30 U/L, platelet count $443 \times 10^{9} / \mathrm{L}$, WBC count $8.30 \times 10^{9} / \mathrm{L}$, and lymphocyte count $2.19 \times 10^{9} / \mathrm{L}$. Antibiotic therapy was discontinued and she was discharged in good conditions. She had no complaints or abnormal physical examination findings at 1-month outpatient follow-up.

\section{Discussion}

Here, we present a case of measles complicated by AAC. Serological confirmation of measles infection is based on a 4 -fold increase in antibody titers between acute and convalescent sera or the detection of measles-specific IgM antibodies in a single serum sample obtained 1 to 2 weeks after the skin rash appears. ${ }^{6}$ In our case, measles was diagnosed based on clinical findings and serological confirmation.

This is the second case of measles-related AAC

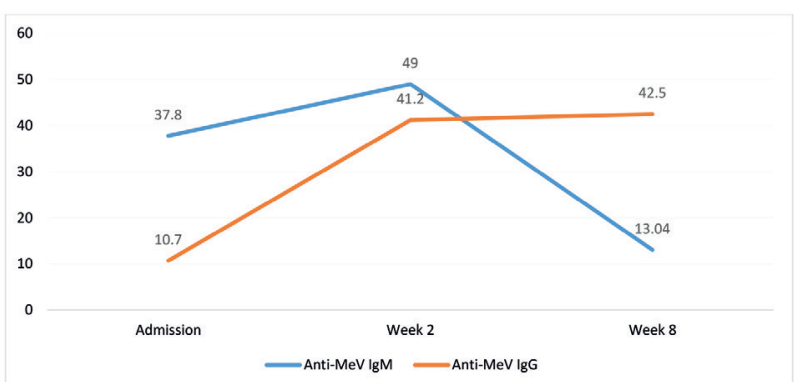

Figure 1. Changes in Anti-measles virus (MeV) IgM and IgG Values on Admission, 2 Weeks, and 8 Weeks $(\mathrm{U} / \mathrm{mL})$. presented in the literature. Similarly to the other case, AAC occurred as a complication during the recovery period. ${ }^{7}$ Measles-associated hepatobiliary complications have been classified into two patterns. The more common is hepatocellular dysfunction, which has been attributed to a viral cytopathic effect. It develops acutely when viral titers peak and ends within a few days after titers fall. The other pattern is characterized by cholestasis and is considered a host-mediated immune response to $\mathrm{MeV}$ infection. It is reported to manifest during recovery and tends to be more symptomatic. However, it has also been suggested that both types of hepatic involvement may be caused by viral cytopathic effects and that cholestasis may only represent a delayed phase of hepatocellular damage that was previously undetected. ${ }^{8}$ Cholestasis resulting from a host-mediated immune response may have been involved in the pathogenesis of AAC during recovery in the present case. In the literature evaluating pneumonia and hepatitis complications during a measles outbreak, increases in ALT and GGT values, as seen in our case, were regarded as an indicator of hepatobiliary involvement. ${ }^{5}$

$\mathrm{AAC}$ is an acute necroinflammatory disease of the gallbladder with a multifactorial pathogenesis. It accounts for approximately $10 \%$ of all cases of acute cholecystitis. ${ }^{9}$ AAC is caused by gallbladder stasis and mucosal ischemia, which leads to a local inflammatory response in the gallbladder wall. Complications such as gallbladder gangrene, empyema, and emphysematous cholecystitis are more severe and develop more rapidly in AAC. Most patients with AAC have multiple risk factors. In some cases, susceptibility to AAC is caused by specific primary infections such as malaria, brucellosis, leptospirosis, Q fever, and dengue fever. ${ }^{10}$ Various viral pathogens associated with AAC include hepatitis A, B, and C, Epstein-Barr virus, and cytomegalovirus. ${ }^{11,12}$ It can also occur in secondary infection of the gallbladder, as in disseminated candidiasis, in chronic biliary carriage of typhoidal and non-typhoidal Salmonella, and in diseases with active diarrhea such as cholera or Campylobacter enteritis. In addition to these, infectious causes such as ascariasis and echinococcal cysts have been reported to cause AAC via obstruction. AAC in patients with acquired immunodeficiency syndrome (AIDS) and other immunosuppressed patients may be associated with opportunistic infections such as microsporidia, Cryptosporidium, or cytomegalovirus. ${ }^{10}$

Patients with overlooked AAC require urgent treatment due to the risk of complications with high mortality, such as gallbladder gangrene and perforation. ${ }^{9}$ The treatment of AAC includes supportive therapies such as intravenous fluid replacement, pain control, as well as initiating antibiotic therapy and cholecystectomy or gallbladder drainage as definitive treatment. Patients who undergo cholecystectomy recover rapidly, generally within 24 hours. ${ }^{13}$ Unlike the other similar case presented in the literature, we managed our case with antibiotic therapy 
and surgery because the patient's cholecystitis symptoms did not resolve immediately.

It is apparent that measles, a vaccine-preventable disease, will become more prevalent due to the surge in anti-vaccine sentiment in recent years. Gallbladder US should be considered in patients with measles and right upper quadrant tenderness. Due to our patient's lack of immediate clinical response and the high complication rate of AAC, we performed surgery and administered antibiotic therapy. This case report aims to raise awareness of AAC as a rare complication associated with measles and demonstrate its effective treatment with antibiotic and surgical treatment.

\section{Authors' Contribution}

EFK and OK participated in the planning, execution, or analysis of the study, contributed to the design and implementation of the study and read and approved the final submitted version. EFK supervised the conduct and analyses of this study.

\section{Conflict of Interest Disclosures}

The authors declare that they have no conflict of interest.

\section{Ethical Statement}

The study was approved by the Clinical Studies Ethics Committee of Erzurum Regional Training and Research Hospital (no:2021/04-76, date:15 Feb 2021).

\section{References}

1. Perry RT, Halsey NA. The clinical significance of measles: a review. J Infect Dis. 2004;189 Suppl 1:S4-16. doi: 10.1086/377712.

2. Gershon AA. Measles virus (rubeola). In: Bennett JE, Dolin R, Blaser MJ, eds. Mandell, Douglas, and Bennett's Principles and Practice of Infectious Diseases. 9th ed. Philadelphia, PA: Elsevier; 2020. p. 2110-6.

3. Xerri T, Darmanin N, Zammit MA, Fsadni C. Complications of measles: a case series. BMJ Case Rep. 2020;13(2). doi: 10.1136/bcr-2019-232408.

4. Tishler M, Abramon AL. Hepatitis and measles. Arch Intern Med. 1983;143(12):2333-4.

5. Kakoullis L, Sampsonas F, Giannopoulou E, Kalogeropoulou C, Papachristodoulou E, Tsiamita M, et al. Measlesassociated pneumonia and hepatitis during the measles outbreak of 2018. Int J Clin Pract. 2020;74(2):e13430. doi: 10.1111/ijcp.13430.

6. Brooks GF, Carroll KC, Butel JN, Morse SA, Mietzer TA. Rubella (German measles) virus. In: Jawetz, Melnick \& Adelberg's Medical Microbiology. 26th ed. USA: McGrawHill; 2013. p. 607-12.

7. Clerckx C, Yombi JC, Belkhir L, Vandercam B. O measlesassociated acute acalculous cholecystitis. Adv Infect Dis. 2012;2(4):97-9. doi: 10.4236/aid.2012.24016.

8. Khatib R, Siddique M, Abbass M. Measles associated hepatobiliary disease: an overview. Infection. 1993;21(2):112-4. doi: 10.1007/bf01710744.

9. Kalliafas S, Ziegler DW, Flancbaum L, Choban PS. Acute acalculous cholecystitis: incidence, risk factors, diagnosis, and outcome. Am Surg. 1998;64(5):471-5.

10. Barie PS, Eachempati SR. Acute acalculous cholecystitis. Gastroenterol Clin North Am. 2010;39(2):343-57, x. doi: 10.1016/j.gtc.2010.02.012.

11. Iaria C, Arena L, Di Maio G, Fracassi MG, Leonardi MS, Famulari C, et al. Acute acalculous cholecystitis during the course of primary Epstein-Barr virus infection: a new case and a review of the literature. Int J Infect Dis. 2008;12(4):391-5. doi: 10.1016/j.ijid.2007.10.005.

12. Omar A, Osman M, Bonnet G, Ghamri N. Acute acalculous cholecystitis caused by hepatitis C: a rare case report. Int J Surg Case Rep. 2016;19:78-81. doi: 10.1016/j. ijscr.2015.12.020.

13. Huffman JL, Schenker S. Acute acalculous cholecystitis: a review. Clin Gastroenterol Hepatol. 2010;8(1):15-22. doi: 10.1016/j.cgh.2009.08.034. 